\title{
HIPOMETABOLISMO CEREBRAL EM PACIENTES COM ESCLEROSE MESIAL TEMPORAL DEMONSTRADO PELO FDG-PET
}

\author{
PAULO S. DUARTE* HONGMING ZHUANG**, DAVID KING***, ABASS ALAVI**
}

\begin{abstract}
RESUMO - O objetivo deste estudo foi avaliar a extensão do hipometabolismo cerebral em pacientes com esclerose mesial temporal (EMT). Método: Este estudo retrospectivo incluiu 21 pacientes que apresentavam epilepsia parcial complexa refrataria à terapia e que foram selecionados para cirurgia após análise extensa que incluía: EEG de superfície e estudos de neuroimagem (PET, SPECT e ressonância magnética). Todos os pacientes foram submetidos a intervenção cirúrgica e tiveram confirmação histológica de EMT. Uma análise semi-quantitativa foi realizada, utilizando regiões de interesse (ROIs) nas seguintes estruturas: lobos frontais, parietais e occipitais, gânglios da base, tálamos, cerebelo e três diferentes regiões nos lobos temporais, que compreendiam o córtex medial, inferior e lateral. Um índice de assimetria (IA) foi calculado, comparando as contagens por pixel nas estruturas homólogas em ambos os hemisférios cerebrais. Os IAs das diferentes estruturas foram então correlacionados. Resultados: Uma correlação significativa foi demonstrada entre os IAs do córtex medial dos lobos temporais e aqueles dos lobos frontais, dos lobos parietais, dos gânglios da base e dos tálamos $(\mathrm{r}=0,72$, $0,62,0,47$ e 0,47 respectivamente com $p<0,05$ ). Foi demonstrada correlação altamente significativa dos IAs das 3 regiões do lobo temporal entre si (chegando a 0,86 entre os IAs das regiões mediais do lobo temporal e os IAs das regiões inferiores). Conclusão: Esses dados indicam que o hipometabolismo se estende além do foco epiléptico no lobo temporal em pacientes com epilepsia parcial complexa relacionada a EMT. O metabolismo na porção medial do lobo temporal é mais correlacionado com o metabolismo no lobo frontal do que com aquele de outras estruturas cerebrais externas aos lobos temporais. Os mecanismos fisiopatológicos envolvidos no hipometabolismo continuam controversos.
\end{abstract}

PALAVRAS-CHAVE: FDG, PET, esclerose mesial temporal, epilepsia.

\section{Brain hypometabolism in patients with mesial-temporal sclerosis demonstrated by FDG-PET}

ABSTRACT - The purpose of this study was to evaluate the extent of brain hypometabolism in patients with mesial-temporal sclerosis (MTS). Method: This retrospective study included 21 patients who had medically refractory complex partial seizures and were selected for surgical therapy after a comprehensive evaluation which included surface EEG recordings and neuroimaging studies (PET, SPECT and MRI). All patients were subjected to surgical intervention and had an histopathological confirmation of MTS. A semi-quantitative analysis of the PET images was performed using regions of interest in the following structures: frontal, parietal and occipital lobes, basal ganglia, thalami, cerebellum and three different regions in the temporal lobes, which included medial, inferior and lateral cortices. An asymmetry index (AI) was calculated, comparing the counts per pixel in the homologous structures in both brain hemispheres. The AI of the different structures were then correlated. Results: A significant correlation was demonstrated between the AI of the medial temporal cortices and the frontal lobe, parietal lobe, basal ganglia and thalami $(r=0.72,0.62,0.47$ and 0.47 respectively with $\mathrm{p}<0.05)$. Within the temporal lobe, highly significant correlations were demonstrated among the structures (as high as 0.86 between temporo-medial and temporo-inferior). Conclusion: These data indicated that hypometabolism extends beyond the epileptogenic focus in the temporal lobe in patients with complex partial seizure related to

*Divisão de Medicina Nuclear, Laboratório Fleury, São Paulo, Brasil; **Divisão de Medicina Nuclear, Hospital da Universidade da Pensilvânia Filadélfia, Pensilvânia, USA; ***California Pacific Medical Center, São Francisco, Califórnia, USA. Dr. Hongming Zhuang foi parcialmente apoiado pelo NIH training grant CA65442-02. Aceite: 23-junho-2000.

Dr. Paulo Schiavom Duarte - Rua Cincinato Braga 282 - Setor de Medicina Nuclear, Laboratório Fleury 01333-910 São Paulo SP - Brasil. Fax 284 1352. E-mail: paulo.duarte@fleury.com.br 
MTS. The metabolism in the medial portion of the temporal lobe is more correlated with the metabolism in the frontal lobe than with those of the others brain structures outside the temporal lobe. The pathophysiological mechanisms of hypometabolism remain controversial.

KEY WORDS: FDG, PET, mesial-temporal sclerosis, epilepsy.

O uso da tomografia por emissão de pósitrons (PET) utilizando a ${ }^{18} \mathrm{~F}$-2-deoxiglicose (FDG) um análogo radioativo da glicose - na fase interictal, é método bem estabelecido para a localização do foco epiléptico em pacientes com epilepsia parcial complexa e que se suspeita ter sua origem em um dos lobos temporais ${ }^{1-3}$. O hipometabolismo observado em um dos lobos temporais é considerado típico para a presença de foco epiléptico nessa localização e vem a complementar as informações obtidas pelos outros exames utilizados na definição de um diagnóstico final de esclerose mesial temporal $(\mathrm{EMT})^{1,2}$.

EMT é um diagnóstico histopatológico caracterizado por perda neuronal e gliose no hipocampo ${ }^{4,5}$. Esta anormalidade estrutural do lobo temporal é frequentemente associada com quadros de epilepsia parcial complexa $a^{6-10}$. O diagnóstico acurado dessa patologia é muito importante, visto que a lobectomia temporal resulta em parada ou redução das convulsões nesses pacientes ${ }^{7,10-12}$. Por isso, o estudo com FDG-PET inter-ictal tem sido usado rotineiramente, quando disponível, na análise de pacientes com suspeita de EMT.

A zona hipometabólica detectada nos estudos de FDG-PET é observada na área de alteração histológica e nas suas proximidades. No entanto, o hipometabolismo não é restrito a estas áreas e frequentemente se estende para outras regiões cerebrais poupadas de anormalidades macroscópicas ou microscópicas ${ }^{1,3,13-15}$. Vários estudos em pacientes com EMT descrevem a extensão do hipometabolismo para outras estruturas cerebrais externa ao lobo temporal acometido, sem uma correspondente lesão anatômica ou histopatológica observada na análise dos exames radiológicos e anatômo-patológicos ${ }^{14,16-20}$. Há considerável número de estudos que abordam este assunto. A maioria deles somente descreve estas alterações ou tenta correlacionar estes achados com os sintomas neuropsiquiátricos do paciente e com as características da crise convulsiva. Há pouca informação na literatura sobre quais estruturas cerebrais são mais frequentemente afetadas e sobre a correlação entre o grau de hipometabolismo no lobo temporal e o das outras regiões cerebrais. Neste estudo, o grau de assimetria do metabolismo entre as porções mediais dos lobos temporais em pacientes com EMT e epilepsia parcial complexa, foi correlacionado com o grau de assimetria presente no metabolismo das outras regiões cerebrais, a fim de determinar a existência e a extensão do hipometabolismo nas estruturas próximas e distantes da região acometida pela lesão histopatológica.

O objetivo deste estudo é avaliar a extensão do hipometabolismo cerebral em pacientes com EMT e epilepsia parcial complexa, utilizando o FDG-PET.

\section{MÉTODO}

\section{Pacientes}

Este estudo retrospectivo incluiu 21 pacientes (9 homens, 12 mulheres; com idade variando de 18 a 44 anos; idade média 30,5 anos; 18 destros e 3 canhotos) (Tabela 1). Todos tinham epilepsia parcial complexa refratária à terapia, e foram selecionados para tratamento cirúrgico após avaliação extensa que incluía EEG de superfície e estudos de neuroimagem (PET, SPECT e ressonância magnética). Todos foram submetidos a lobectomia temporal do tipo standard baseada nos resultados obtidos nesses estudos, e tiveram confirmação histopatológica de EMT. Pacientes com alterações cerebrais externas ao lobo temporal acometido, detectadas na ressonância magnética, foram excluídos deste estudo.

\section{Métodos}

Um estudo de FDG-PET interictal foi realizado antes da cirurgia. Nem os pacientes, nem os familiares referiram episódios de convulsão em um período inferior a 6 horas da realização do exame. Os pacientes foram injetados com ${ }^{18} \mathrm{~F}$-2-deoxiglicose $(1.3 \mathrm{MBq}-4.2 \mathrm{MBq} / \mathrm{Kg})$ em um quarto escuro, com os olhos abertos e os 
Tabela 1. Informações dos pacientes.

\begin{tabular}{|c|c|c|c|c|c|c|}
\hline Paciente & $\begin{array}{l}\text { Idade } \\
\text { (anos) }\end{array}$ & Sexo & Dominância & Idade $1^{\mathrm{a}}$ crise & $\begin{array}{l}\text { Idade de recorr. } \\
\text { da crise (anos) }\end{array}$ & tipo de crise \\
\hline 1 & 43 & M & $\mathrm{E}$ & 25 anos & 25 & $\mathrm{CPC}$ \\
\hline 2 & 39 & F & $\mathrm{D}$ & 4 anos & 13 & $\mathrm{CPC}$ \\
\hline 3 & 18 & M & $\mathrm{D}$ & 7 anos & 7 & $\mathrm{CPC}$ \\
\hline 4 & 30 & F & $\mathrm{D}$ & 18 anos & 18 & $\mathrm{CPC}$ \\
\hline 5 & 34 & $\mathrm{~F}$ & $\mathrm{D}$ & 8 anos & 8 & $\mathrm{CPC}$ \\
\hline 6 & 36 & $\mathrm{~F}$ & $\mathrm{D}$ & 18 anos & 18 & $\mathrm{CPC}$ \\
\hline 7 & 25 & $\mathrm{~F}$ & $\mathrm{E}$ & 5 anos & 5 & $\mathrm{CPC}$ \\
\hline 8 & 30 & $\mathrm{~F}$ & $\mathrm{D}$ & 2 anos & 10 & $\mathrm{CPC}$ \\
\hline 9 & 39 & M & $\mathrm{D}$ & 2 anos & 2 & $\mathrm{CPC}$ \\
\hline 10 & 21 & M & $\mathrm{D}$ & 14 anos & 14 & $\mathrm{CPC}$ \\
\hline 11 & 36 & M & $\mathrm{D}$ & 6 meses & 17 & $\mathrm{CPC}$ \\
\hline 12 & 43 & F & $\mathrm{D}$ & 6 anos & 6 & $\mathrm{CPC}$ \\
\hline 13 & 35 & M & $\mathrm{D}$ & 4 anos & 7 & $\mathrm{CPC}$ \\
\hline 14 & 44 & M & $\mathrm{D}$ & 3 anos & 3 & $\mathrm{CPC}$ \\
\hline 15 & 43 & F & $\mathrm{E}$ & 32 anos & 32 & $\mathrm{CPC}$ \\
\hline 16 & 39 & F & $\mathrm{D}$ & 1 ano & 6 & $\mathrm{CPC}$ \\
\hline 17 & 34 & M & $\mathrm{D}$ & 3 anos & 6 & $\mathrm{CPC}$ \\
\hline 18 & 39 & $\mathrm{~F}$ & $\mathrm{D}$ & 11 meses & 30 & $\mathrm{CPC}$ \\
\hline 19 & 32 & $\mathrm{~F}$ & $\mathrm{D}$ & 1 ano & 1 & $\mathrm{CPC}$ \\
\hline 20 & 21 & M & $\mathrm{D}$ & 18 anos & 18 & $\mathrm{CPC}$ \\
\hline 21 & 39 & F & D & 4 anos & 4 & CPC \\
\hline
\end{tabular}

$\mathrm{CPC}$, crise parcial complexa.

ouvidos não oclusos. Eles foram observados por tecnólogo experiente no período após a injeção até a realização das imagens (aproximadamente 60 minutos), a fim de excluir a possibilidade de crises nesta fase do estudo. Os pacientes foram mantidos durante todo o tempo com o seu esquema de medicação usual. As imagens foram adquiridas usando o HEAD PENN PET ${ }^{21}$ ou o PENN PET $240 \mathrm{H}^{22}$ scanners. Ambos os instrumentos utilizam cristais de iodeto de sódio ( $\mathrm{NaI}(\mathrm{Tl}))$ para a detecção de raios $\gamma$ e operam em um modo tridimensional sem septos. O tamanho do voxel para ambos os sistemas é de $2 \times 2 \times 2 \mathrm{~mm}$ em todos os 3 planos e a resolução espacial é 5,5 $\mathrm{mm}$ e 3,7 mm para o PENN PET 240H e HEAD PENN PET, respectivamente. As imagens foram processadas usando o programa PETView em uma estação de trabalho Sun. A ressônancia magnética foi realizada em equipamento de 1.5 Tesla com SPGR, espessura de corte de $1.5 \mathrm{~mm}$, com imagens obtidas em posição coronal obliqua nas sequências T1 e FLAIR

\section{Análise dos dados}

Para realizarmos análises semi-quantitativas, utilizamos imagens não filtradas porém corrigidas para atenuação. Após uma reorientação obliqua, foram realizados cortes ( $2 \mathrm{~mm}$ de espessura) perpendicularmente ao maior eixo do lobo temporal (cortes coronais otimizados para uma melhor avaliação do lobo temporal). Seis regiões de interesse (ROI) foram desenhadas por um único investigador em cada um de 19 cortes do lobo temporal, se iniciando a $1 \mathrm{~cm}$ do polo anterior e se estendendo posteriormente. Estes ROIs dividiram os lobos temporais em três partes de cada lado (lateral, medial e inferior) (Fig 1). Isso resultou em 6 grupos de regiões a saber: temporal lateral esquerda, temporal lateral direita, temporal inferior esquerda, temporal inferior direita, temporal medial esquerda e temporal medial direita.

A contagem total de eventos radioativos e o número de total de pixels em cada região foram somados com aqueles das regiões correspondentes em todos os 19 cortes feitos nos lobos temporais. Em seguida, a soma 


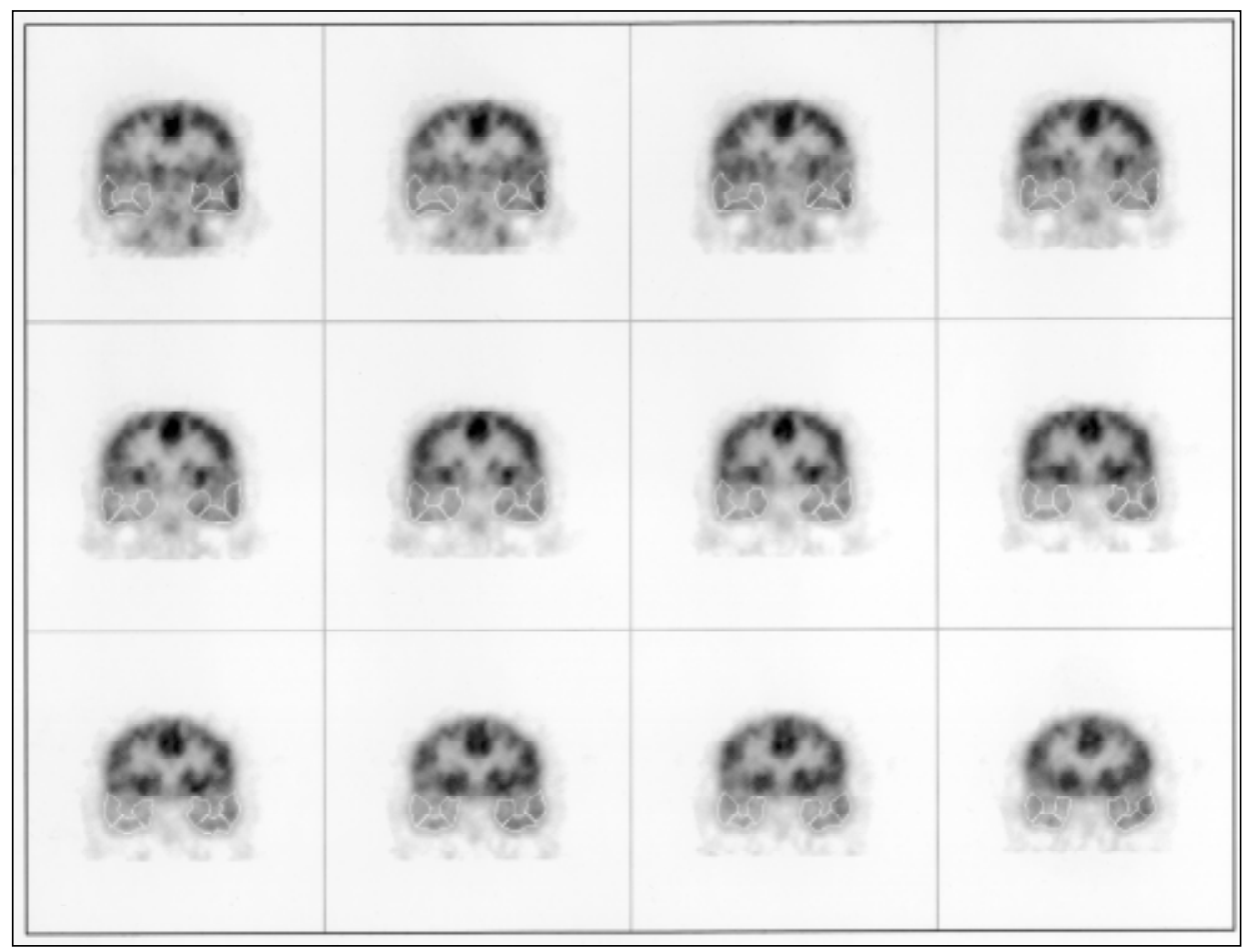

Fig 1. Cortes coronais do cérebro perpendiculares ao longo eixo do lobo temporal mostrando a divisão de cada lobo temporal nas suas porções lateral, medial e inferior.

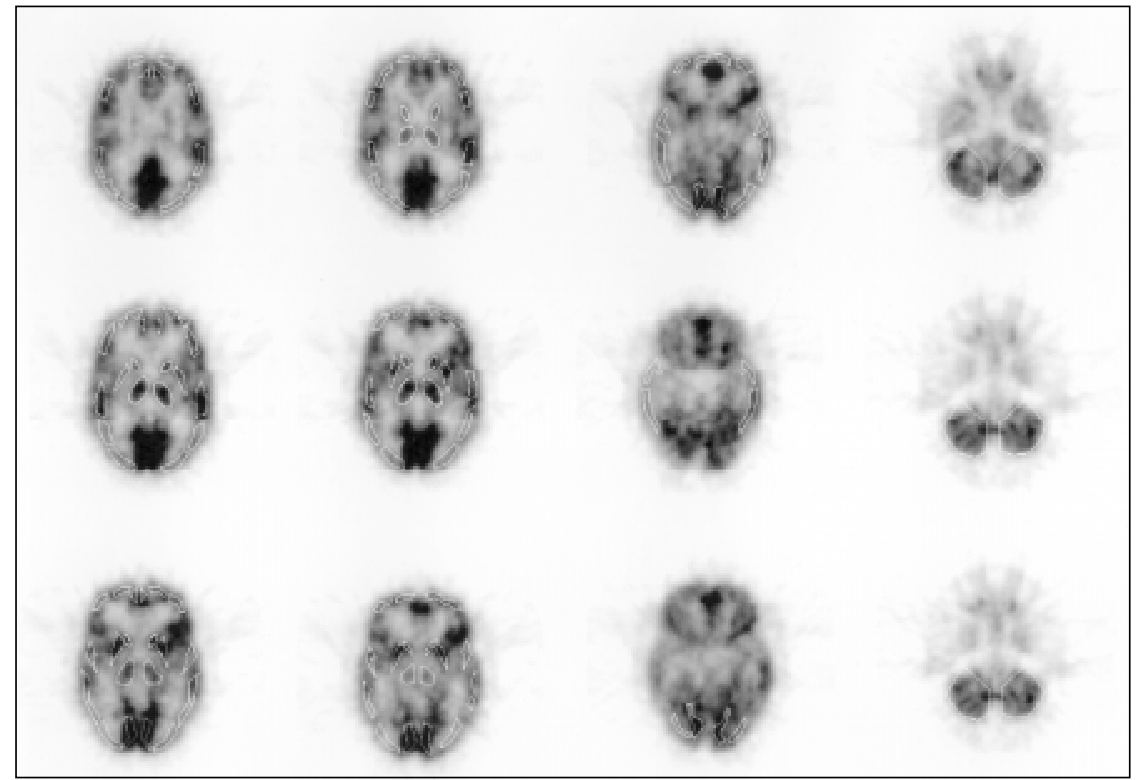

Fig 2. Cortes transversais do cérebro (12 níveis representados) mostrando uma máscara de regiões de interesse sobrepostas às áreas a serem analisadas. Apesar de observarmos regiões de interesse posicionadas nos lobos temporais, elas não foram utilizadas para análise. 
Tabela 2. Índices de assimetria de todas as regiões calculados para cada paciente.

\begin{tabular}{cccccccccc}
\hline Paciente & Tálamo Cerebelo & $\begin{array}{c}\text { Gânglios } \\
\text { da base }\end{array}$ & Frontal & Parietal & Occipital & $\begin{array}{c}\text { Temporal } \\
\text { L }\end{array}$ & $\begin{array}{c}\text { Temporal } \\
\text { I }\end{array}$ & $\begin{array}{c}\text { Temporal } \\
\text { M }\end{array}$ \\
\hline 1 & 0,1 & $-1,0$ & $-3,0$ & $-5,3$ & $-4,3$ & $-1,5$ & $-13,8$ & $-10,8$ & $-4,0$ \\
2 & $-1,1$ & 0,5 & 0,3 & $-0,9$ & $-1,6$ & 0,1 & 0,8 & $-0,7$ & $-4,6$ \\
3 & $-1,7$ & $-6,2$ & $-1,6$ & $-3,6$ & 3,9 & $-1,9$ & $-18,9$ & $-16,9$ & $-3,9$ \\
4 & 0,5 & $-8,2$ & 0,6 & $-0,8$ & $-1,0$ & $-2,8$ & $-9,2$ & $-5,3$ & $-4,7$ \\
5 & 1,9 & $-7,8$ & 1,8 & 5,3 & 4,3 & 5,6 & 26,6 & 25,9 & 14,1 \\
6 & $-3,0$ & 2,4 & 0,9 & 3,7 & 3,0 & 4,6 & 27,0 & 23,1 & 16,1 \\
7 & 5,1 & $-6,6$ & 4,5 & $-0,9$ & $-2,7$ & $-2,2$ & 3,3 & 2,5 & 0,5 \\
8 & 1,7 & 0,3 & 6,6 & 8,2 & 6,4 & 5,3 & 39,7 & 42,9 & 12,7 \\
9 & $-2,6$ & 4,3 & 0,6 & $-4,6$ & $-6,0$ & 0,5 & $-19,7$ & $-22,1$ & $-18,2$ \\
10 & $-1,5$ & $-1,4$ & 1,8 & 0,7 & 1,1 & 1,0 & $-4,9$ & 1,3 & 3,1 \\
11 & $-0,4$ & $-5,1$ & 2,6 & $-4,4$ & 0,0 & $-7,1$ & $-5,1$ & $-9,5$ & $-10,5$ \\
12 & 7,8 & $-1,3$ & 2,9 & $-4,9$ & $-1,0$ & 2,6 & $-4,8$ & 5,0 & 9,6 \\
13 & $-1,8$ & $-3,7$ & $-4,4$ & $-4,9$ & $-5,1$ & $-5,7$ & $-14,7$ & $-11,3$ & $-1,1$ \\
14 & 2,5 & 0,0 & 12,3 & 8,5 & 13,4 & 3,6 & 8,3 & 10,0 & 3,5 \\
15 & 3,5 & $-3,3$ & 5,3 & 1,2 & 5,7 & $-0,8$ & 13,2 & 16,2 & $-0,5$ \\
16 & 4,6 & $-2,8$ & $-1,3$ & $-5,1$ & $-0,9$ & $-2,0$ & $-11,3$ & $-5,6$ & $-4,5$ \\
17 & $-6,6$ & $-1,6$ & $-4,2$ & $-11,3$ & $-1,9$ & 7,6 & $-14,3$ & $-14,6$ & $-11,2$ \\
18 & 7,8 & 0,0 & 0,5 & 4,0 & 3,7 & 4,7 & 8,7 & 16,1 & 3,2 \\
19 & $-1,9$ & $-0,3$ & $-3,1$ & $-7,4$ & $-7,2$ & 6,1 & $-15,4$ & $-8,7$ & $-15,5$ \\
20 & $-0,9$ & $-5,5$ & 2,4 & 0,5 & 3,4 & 1,4 & 12,4 & 16,1 & 9,3 \\
21 & $-10,9$ & 2,8 & $-2,2$ & $-4,9$ & $-3,7$ & $-6,1$ & $-18,6$ & $-21,3$ & $-14,8$ \\
Média* & 3,2 & 3,1 & 3,0 & 4,3 & 3,8 & 3,5 & 13,8 & 13,6 & 7,9 \\
Desv & 2,9 & 2,6 & 2,7 & 2,9 & 3,0 & 2,3 & 9,2 & 9,9 & 5,7 \\
\hline
\end{tabular}

*As médias foram calculadas a partir dos valores absolutos.

das contagens dos eventos radioativos em cada grupo de regiões foi dividida pela soma do número de pixels dentro do mesmo grupo de regiões para obtermos a contagem média por pixel de cada grupo de regiões.

As contagens médias por pixel dos grupos de regiões homólogos nos lobos temporais direito e a esquerdo foram comparados usando um índice de assimetria (IA), calculado através da equação: $[(R-L) /(R+L)] \times 200$ (Tabela 2). Onde R é a contagem média por pixel no grupo de regiões do lado direito e L é a contagem média por pixel no grupo de regiões homólogo no lobo temporal esquerdo.

A análise do metabolismo nas estruturas externas aos lobos temporais foi realizada nos cortes transversais do estudo de FDG-PET cerebral, utilizando-se de uma máscara com regiões de interesse ${ }^{23}$. Essa máscara foi modificada para os propósitos deste estudo, de forma que, somente algumas das regiões (176 ROIs em 16 níveis de corte) foram usadas para analisar as imagens (Fig 2). As seguintes estruturas cerebrais foram analisadas: lobos frontais (82 ROIs), lobos parietais (32 ROIs), lobos occipitais (28 ROIs), cerebelo (6 ROIs), tálamos (10 ROIs) e gânglios da base (18 ROIs). De forma similar ao realizado com as regiões nos lobos temporais, a contagem total de eventos radioativos e o número de total de pixels nos ROIs representando a mesma estrutura cerebral no mesmo hemisfério foram somados. Esses valores foram usados para calcular o número de contagens de eventos radioativos por pixel nesses grupos de regiões. A contagem de eventos radioativos por pixel no grupo de ROIs representando estruturas cerebrais homólogas nos dois hemisférios foram usadas para calcular IAs (Tabela 2), como descrito acima. Os índices de assimetria dessas estruturas cerebrais foram correlacionados com aquele da região medial do lobo temporal. Os índices de assimetria das regiões temporais também foram correlacionados entre si (Tabela 3). O coeficiente de correlação ( $r$ ) entre os IAs foi utilizado para estabelecer as relações entre o grau de assimetria no metabolismo das diferentes regiões cerebrais. 
Tabela 3. Coeficientes de correlação entre todas as estruturas cerebrais analisadas.

Tálamo Cerebelo Gânglios Frontal Parietal Occipital da base
Temporal

L $\quad \mathrm{I} \quad \mathrm{M}$

\begin{tabular}{lccccccccc}
\hline Tálamo & 1,00 & & & & & & & & \\
Cerebelo & $-0,30$ & 1,00 & & & & & & \\
Gânglios da base & 0,47 & $-0,03$ & 1,00 & & & & & \\
Frontal & 0,39 & $-0,02$ & 0,76 & 1,00 & & & & \\
Parietal & 0,34 & $-0,14$ & 0,77 & 0,80 & 1,00 & & & & \\
Occipital & 0,17 & 0,24 & 0,18 & 0,30 & 0,31 & 1,00 & & & \\
Temporal L & 0,36 & $-0,05$ & 0,61 & 0,84 & 0,67 & 0,47 & 1,00 & & \\
Temporal I & 0,48 & $-0,08$ & 0,60 & 0,83 & 0,67 & 0,52 & 0,97 & 1,00 & \\
Temporal M & 0,47 & $-0,21$ & 0,47 & 0,72 & 0,62 & 0,38 & 0,82 & 0,86 & 1,00 \\
\hline
\end{tabular}

\section{RESULTADOS}

A atividade metabólica da porção medial dos lobos temporais está fortemente correlacionada com aquela das outras porções destes lobos (lateral e inferior), apresentando coeficientes de correlação de 0,86 e 0,82 ( $\mathrm{p}<0,0001$ ), respectivamente. Há, também, correlação significativa entre o metabolismo na porção medial dos lobos temporais e aquele dos lobos frontais $(r=0,72, p<0,001)$, lobos parietais $(\mathrm{r}=0,62, \mathrm{p}=0,002)$, tálamos $(\mathrm{r}=0,47, \mathrm{p}=0,03)$ e gânglios da base $(\mathrm{r}=0,47, \mathrm{p}=$ $0,03)$. Nenhuma correlação significativa foi observada entre a atividade metabólica na porção medial dos lobos temporais e aquela do cerebelo $(r=-0,21, p=0,36)$ e lobos occipitais $(r=0,38, p=0,08)$.

\section{DISCUSSÃO}

Neste estudo, a assimetria do metabolismo nas porções mediais dos lobos temporais parece estar significativamente correlacionada com aquela das outras estruturas cerebrais analisadas com exceção do cerebelo e dos lobos occipitais. A importância dessa observação consiste em que as alterações metabólicas da patologia em questão afetam, não somente as regiões circunvizinhas à lesão histopatológica, mas também se estendem para outras estruturas cerebrais distantes do foco epiléptico no lobo temporal ${ }^{1,14,15}$. As maiores correlações observadas entre o metabolismo na porção medial do lobo temporal e aquele das estruturas extra temporais foram observadas com os lobos frontais e parietais. Este achado é interessante, visto que estudos prévios sugerem que o tálamo deva ser a região extratemporal mais afetada, devido a rica conexão entre as duas estruturas ${ }^{14,17,19,20}$.

A razão para a presença de hipometabolismo nas regiões cerebrais externas ao lobo temporal em pacientes com EMT não é compreendida por completo. Henry et al. sugerem que a redução na concentração celular na porção medial do lobo temporal e a diaschisis não são os principais fatores responsáveis por este hipometabolismo. Outros mecanismos, como alterações funcionais causadas por episódios convulsivos de longa duração, perda neuronal no córtex e alterações na densidade das sinapses, poderiam ser alguns dos mecanismos envolvidos ${ }^{15}$. O'Brien et al. mostraram que não há correlação entre o grau de hipometabolismo nas porções mediais ou laterais do lobo temporal e o grau de atrofia hipocampal estimada na análise volumétrica do lobo temporal, realizada pela ressonância magnética, e também concluíram que a redução celular no hipocampo não deva ser o principal mecanismo envolvido no hipometabolismo das regiões cerebrais de pacientes com EMT ${ }^{24}$. Margerison e Corsellis descreveram a presença de perda celular nos tálamos e no córtex de alguns pacientes com EMT e sugerem que esta perda poderia ser a responsável por parte do hipometabolismo 
observado nestes pacientes ${ }^{25}$. Logo, a combinação de uma série de mecanismos como: perda celular ${ }^{25}$, diminuição da atividade aferente ${ }^{20}$, alterações funcionais decorrentes de episódios convulsivos longos e frequentes ${ }^{18}$, e o efeito de medicações anti-convulsivas ${ }^{26-29}$ é uma possível explicação para o hipometabolismo.

Independentemente dos mecanismos fisiopatológicos envolvidos no hipometabolismo cerebral neste tipo de patologia, os lobos frontais e outras estruturas cerebrais apresentam algum grau de alteração funcional. Este achado vem a reforçar publicações prévias que constatam que na EMT , o metabolismo cerebral, na maior parte do hemisfério ipsilateral à lesão, apresenta algum grau de diminuição ${ }^{14,18}$.

Esta informação pode ser útil na compreensão das consequências da EMT para o funcionamento cognitivo dos pacientes acometidos por esta patologia. Atualmente, a epilepsia é considerada uma doença que afeta o metabolismo cerebral do paciente não só durante as crises, mas também no período intercrises ${ }^{30} \mathrm{e}$, provavelmente, as alterações metabólicas não estão restritas ao lobo temporal acometido, justificando déficits cognitivos que esses pacientes possam ter no período intercrises.

\section{Conclusão}

Esses dados indicam que o hipometabolismo se estende além do foco epiléptico no lobo temporal em pacientes com epilepsia parcial complexa relacionada à EMT. O metabolismo na porção medial do lobo temporal parece estar mais correlacionado com o metabolismo no lobo frontal do que com aquele das outras estruturas cerebrais externas aos lobos temporais. Os mecanismos fisiopatológicos envolvidos neste hipometabolismo ainda não são completamente compreendidos. Futuros estudos são necessários para esclarecer estes mecanismos.

Agradecimentos - Os autores agradecem a Michael Pourdehnad, Eleathea Barraclough, Olivier Couturier e Flávia Aldighieri pelas suas análises críticas deste estudo.

\section{REFERÊNCIAS}

1. Engel J Jr, Brown WJ, Kuhl DE, Phelps ME, Mazziotta JC, Crandall PH. Pathological findings underlying focal temporal lobe hypometabolism in partial epilepsy. An Neurol 1982;12:518-528.

2. Mazziotta JC, Engel J Jr. The use and impact of positron computed tomography scanning in epilepsy. Epilepsia 1984;25 (Suppl 2):S86-104.

3. Sackellares JC, Siegel GJ, Abou-Khalil BW, et al.. Differences between lateral and mesial temporal metabolism interictally in epilepsy of mesial temporal origin. Neurology 1990;40:1420-1426.

4. Dam AM. Consequences of severe epilepsy: neuropathological aspects. Acta Neurol Scand 1988(Suppl);117:24-27.

5. Sagar HJ, Oxbury JM. Hippocampal neuron loss in temporal lobe epilepsy: correlation with early childhood convulsions. An Neurol 1987;22:334-340.

6. Dam AM. Epilepsy and neuron loss in the hippocampus. Epilepsia 1980;21:617-629.

7. Falconer MA. The significance of mesial temporal sclerosis (Ammon's horn sclerosis) in epilepsy. Guys.Hospital. Reports 1968;117:1-12.

8. Gates JR, Cruz-Rodriguez R. Mesial temporal sclerosis: pathogenesis, diagnosis, and management. Epilepsia 1990;31(Suppl 3):55-66.

9. Spencer DD, Spencer SS. Hippocampal resections and the use of human tissue in defining temporal lobe epilepsy syndromes. Hippocampus 1994;4:243-249.

10. Spencer SS, Spencer DD. Entorhinal-hippocampal interactions in medial temporal lobe epilepsy. Epilepsia 1994;35:721-727.

11. Duncan JS, Sagar HJ. Seizure characteristics, pathology, and outcome after temporal lobectomy. Neurology 1987;37:405-409.

12. Falconer MA, Taylor DC. Surgical treatment of drug-resistant epilepsy due to mesial temporal sclerosis: etiology and significance. Arch Neurology 1968;19:353-361.

13. Abou-Khalil BW, Siegel GJ, Sackellares JC, Gilman S, Hichwa R, Marshall R. Positron emission tomography studies of cerebral glucose metabolism in chronic partial epilepsy. An Neurol 1987;22:480-486.

14. Henry TR, Mazziotta JC, Engel J Jr. Interictal metabolic anatomy of mesial temporal lobe epilepsy. Arc Neurol 1993;50:582-589.

15. Henry TR, Babb TL, Engel J Jr, Mazziotta JC, Phelps ME., Crandall PH. Hippocampal neuronal loss and regional hypometabolism in temporal lobe epilepsy. Ann Neurol 1994;36:925-927.

16. Arnold S, Schlaug G, Niemann H, et al.. Topography of interictal glucose hypometabolism in unilateral mesiotemporal epilepsy. Neurology 1996;46:1422-1430.

17. Henry TR, Mazziotta JC, Engel J Jr, et al.. Quantifying interictal metabolic activity in human temporal lobe epilepsy. J Cer Blood Flow Metab1990;10:748-757. 
18. Jokeit H, Seitz RJ, Markowitsch HJ, Neumann N, Witte OW, Ebner A. Prefrontal asymmetric interictal glucose hypometabolism and cognitive impairment in patients with temporal lobe epilepsy. Brain 1997;120:2283-2294.

19. Khan N, Leenders KL, Hajek M, Maguire P, Missimer J, Wieser HG. Thalamic glucose metabolism in temporal lobe epilepsy measured with 18f-fdg positron emission tomography (PET). Epilepsy Res 1997;28:233-243.

20. Sperling MR, Gur RC, Alavi A, et al.. Subcortical metabolic alterations in partial epilepsy. Epilepsia 1990;31:145-155.

21. Karp JS, Muehllehner G. Standards for performance measurements of pet scanners: evaluation with the UGM PENN-PET 240H Scanner. Med Progr Technol 1991;17:173-187.

22. Karp JS, Freifelder R, Geagan MJ, et al. Three-dimensional imaging characteristics of the HEAD PENN-PET Scanner. J Nucl Med 1997;38:636-643.

23. Resnick SM, Karp JS, Turetsky B, Gur RE. Comparison of anatomically-defined versus physiologically-based regional localization: effects on PET-FDG quantitation [published erratum appears in J Nucl Med 1994 Jan;35:26]. J Nucl .Med 1993;34:2201-2207.

24. O'Brien TJ, Newton MR, Cook MJ, et al.. Hippocampal atrophy is not a major determinant of regional hypometabolism in temporal lobe epilepsy. Epilepsia 1997;38:74-80.

25. Margerison JH, Corsellis JA. Epilepsy and the temporal lobes: a clinical, electroencephalographic and neuropathological study of the brain in epilepsy, with particular reference to the temporal lobes. Brain 1966;89:499-530.

26. Theodore WH, Bairamian D, Newmark ME, et al.. Effect of phenytoin on human cerebral glucose metabolism. J Cer. Blood Flow Metab1986;6:315-320.

27. Theodore WH, DiChiro G, Margolin R, Fishbein D, Porter RJ, Brooks RA. Barbiturates reduce human cerebral glucose metabolism. Neurology 1986;36:60-64.

28. Theodore WH. Antiepileptic drugs and cerebral glucose metabolism. Epilepsia 1988;29(Suppl 2):48-55.

29. Theodore WH, Bromfield E, Onorati L. The effect of carbamazepine on cerebral glucose metabolism. Ann Neurol 1989;25:516-520.

30. Savic I, Altshuler L, Baxter L, Engel J Jr. Pattern of interictal hypometabolism in pet scans with fludeoxyglucose f 18 reflects prior seizure types in patients with mesial temporal lobe seizures. Arch Neurol 1997;54:129-136. 